\title{
Joint spatio-temporal modelling of adverse pregnancy outcomes sharing common risk factors at sub-county level in Kenya, 2016-2019
}

Julius Nyerere Odhiambo $1,2,3^{*}$ (D) and Benn Sartorius ${ }^{1,4,5}$

\begin{abstract}
Background: Adverse pregnancy outcomes jointly account for a high proportion of mortality and morbidity among pregnant women and their infants. Furthermore, the burden attributed to adverse pregnancy outcomes remains high and inadequately characterised due to the intricate interplay of its etiology and shared set of important risk factors. This study sought to quantify and map the underlying risk of multiple adverse pregnancy outcomes in Kenya at subcounty level using a shared component space-time modelling framework.
\end{abstract}

Methods: Reported sub-county level adverse pregnancy outcomes count from January 2016 - December 2019 were obtained from the Kenyan District Health Information System. A Bayesian hierarchical spatio-temporal model was used to estimate the joint burden of adverse pregnancy outcomes in space (sub-county) and time (year). To improve the precision of our estimates over time and space, information across the outcomes were combined via the shared and the outcome-specific components using a shared component model with spatio-temporal interactions.

Results: Overall, the total number of adverse outcomes in pregnancy increased by 14.2\% (95\% UI: 14.0-14.5) from 88,816 cases in 2016 to 101,455 cases in 2019. Between 2016 and 2019, the estimated low birth weight rate and the pre-term birth rate were 4.5 (95\% UI: 4.4-4.7) and 2.3 (95\% UI: 2.2-2.5) per 100 live births. The stillbirth and neonatal death rates were estimated to be 18.7 (95\% UI: 18.0-19.4) and 6.9 (95\% UI: 6.4-7.4) per 1000 live births. The magnitude of the spatio-temporal variation attributed to shared risk was high for pre-term births, low birth weight, neonatal deaths, stillbirths and neonatal deaths, respectively. The shared risk patterns were dominant in sub-counties located along the Indian ocean coastline, central and western Kenya.

Conclusions: This study demonstrates the usefulness of a Bayesian joint spatio-temporal shared component model in exploiting specific and shared risk of adverse pregnancy outcomes sub-nationally. By identifying sub-counties with elevated risks and data gaps, our estimates not only assert the need for bolstering maternal health programs in the identified high-risk sub-counties but also provides a baseline against which to assess the progress towards the attainment of Sustainable Development Goals.

Keywords: Mapping, Risk, Low birth weight, Pre-term birth, Stillbirths, Neonatal deaths, Bayesian shared component, Kenya

*Correspondence: nyererejulius7@gmail.com

${ }^{1}$ Discipline of Public Health Medicine, College of Health Sciences, University of KwaZulu-Natal, 2nd Floor George Campbell Building, Howard College Campus, Durban 4001, South Africa

Full list of author information is available at the end of the article

\section{Background}

Adverse pregnancy outcomes (APOs) are a major cause of neonatal mortality and morbidity and affects millions of women of childbearing age worldwide and is a critical global health concern. While large strides have been 
made in tracking progress towards the attainment of global maternal health targets in Sub-Saharan Africa (SSA), the World Health Organization (WHO) estimates that approximately 810 women die each day as a result of preventable causes associated with pregnancy and childbirth [1-4]. Despite the global priority and substantial investment in maternal and child health programs in recent decades [5], the burden of APOs remains unacceptably high in SSA and Southern Asia, with both regions accounting for approximately $86 \%$ of the global maternal deaths [1]. Recent estimates of APOs have been 546 maternal deaths per 100,000 live births, 25.9 neonatal deaths per 1000 live births and 13.9 stillbirths per 1000 total births [6-8]. These sobering trends are mirrored in Kenya, which risks missing out on the 2030 neonatal mortality target of 12 deaths per 1000 live births given its high neonatal mortality rate of approximately 19.6 deaths per 1000 live births and 11.5 low birth weight babies per 100 live births reported in 2018 [9]. To address this growing burden, national initiatives such as free maternity services, elimination of user fees for public primary health care and the beyond zero campaign have been established to improve pregnancy outcomes and increase institutional delivery rates [10].

As a step towards renewing its focus towards maternal and child health programs and achieving global health targets, the United Nations Sustainable Development Goals (SDGs) set ambitious health targets for mothers, infants and children. Target 3.1 seeks to reduce maternal mortality ratio to less than 70 per 100,000 live births. In contrast, target 3.2 aims to end preventable neonatal mortality to 12 per 1000 live births and sustain universal healthcare by 2030 [11]. Moreover, the WHO conceptualised two global initiatives namely; Ending Preventable Maternal Mortality (EPMM) [12] and the Every Newborn Action Plan (ENAP) [13]. These initiatives sought to catalyse global action into addressing disparities in maternal health outcomes among sub-populations. These ambitious targets were further entrenched by two of the six global nutritional targets for 2025 [14], which sought to achieve; a $50 \%$ reduction of maternal anaemia and a $30 \%$ reduction of low birth weight (LBW) as vital components for improving maternal health.

The government of Kenya strives to achieve universal health coverage for its population and alleviate the burden imposed by adverse pregnancy outcomes in women of childbearing age. Mapping sub-county level estimates would provide a greater understanding of the variability in spatio-temporal patterns of adverse pregnancy outcomes, not possible by examination of direct national estimates or by examination of direct county-level estimates [15]. This is crucial for; monitoring progress towards global maternal health priorities and orienting national policies to identify high burden sub-counties and high-risk populations $[11,15,16]$.

Sub-nationally, little is also known about the joint and shared risk estimates of adverse pregnancy outcomes and their association with related health outcomes across the continuum of maternal health care. This may be attributed to the complex interplay of risk factors that remains inadequately characterised in the spatial and temporal domain [17-19]. This may be attributed to the paucity of data on individual pregnancy outcomes and risk factors sub-nationally. This becomes more apparent when data is stratified at policy-relevant spatial scales to accommodate different demographic dimensions. To stabilise estimates, strengthen inference occasioned by data scarcity, we borrow information across adjacent sub-counties, diseases/health outcomes and sub-populations with common risk factors. Previous studies have demonstrated the robustness of Bayesian techniques in jointly mapping two or more diseases/health outcomes with common risk factors by leveraging information among the diseases and across neighbouring geographical units [20, 21]. To the best of our knowledge, little work on Bayesian space-time modelling has been done in maternal health research in Kenya. To quantify the underlying burden and increase epidemiologic interpretability of adverse pregnancy outcomes, we employed a Bayesian hierarchical shared component model that simultaneously accounts for both shared and specific risk components attributed to each pregnancy outcome.

\section{Methods}

\section{Geographic analysis unit}

Administratively Kenya is divided into 47 counties tasked with the implementation of national guidelines and policy. The counties are further subdivided into 290 subcounties which was adopted as the unit of analysis in this study (Fig. 1).

\section{Kenya healthcare system}

Kenya's public health system is divided into six levels ranging from community-based care (level 1) to tertiary hospitals (level 6). Level 1 primarily consists of health promotion and awareness-raising activities at the community level; levels 2-3 include primary health care facilities, including dispensaries and health centres; and levels 4-6 include county and national referral hospitals $[22,23]$. The Kenya government prioritises healthcare as a critical element in its development agenda. The Kenya Health Policy 2014-2030 seeks to achieve universal health coverage by targeting health services to regions and populations in need [16]. Additionally, in seeking to improve the facility delivery rates and reduce pregnancyrelated mortality, the government abolished delivery fees 




Fig. 1 The map of Kenya showing 290 sub-counties (numbered), with the extents of major lakes and the Indian ocean is shown in light blue. The names of counties, sub-counties and their malaria endemicity status are presented in supplementary file 2. (Source: https://data.humdata.org/datas et/ken-administrative-boundaries)

in all public health facilities in 2013 [10]. This led to a significant increase in facility deliveries, with the proportion of reported births increasing from $90.1 \%$ in 2015 to $96.7 \%$ by 2019 [24]. Additionally, the devolved governance system stimulated the need for sub-national monitoring of disease burden trends and control/intervention progress. This led to a steady increase in facility reporting completeness rate (estimated to be above 90\%) on key health metrics. The most recent Demographic and Health Survey (DHS) reports that only $61 \%$ of births between 2009 and 2014 were reported to have occurred in a health facility [25].

\section{Data assembly}

Global maternal and newborn health initiatives, Every Newborn Action Plan and Ending Preventable Maternal Mortality, have identified priority indicators derived from facility-based data as important signals for monitoring progress $[12,26]$. Consequently, study data was extracted from the Kenya District Health Information System 
(DHIS2), provided a unique opportunity/effective basis to examine the sub-national variation of APOs from 2016 to 2019 (Table 1).

\section{Selecting a suitable set variable for APOs risk}

A two-step procedure prominent in mapping exercises $[30,31]$ was used to select the best predictors of adverse pregnancy outcomes based on their associative strength and relevance. First a non-spatial Poisson regression model was used to test the bivariate association between APOs and related risk factors. To identify the strength of each candidate covariate as a predictor and to identify the best subset of predictors for each outcome respectively. The Wald's $p$-value, the goodness of fit statistics and the associated credible interval were then assessed. Non-collinear covariates significant at a $p$-value of $<0.05$ were then included in subsequent analyses. (Supplementary file 2).

\section{Joint spatio-temporal shared component model}

The Bayesian hierarchical spatio-temporal shared component model was used to simultaneously quantify the burden of four APOs often characterised by low counts. To improve the precision of estimates over time and space, information (similarities and differences) across the APOs was combined via the shared and the outcomespecific components using a shared component model with spatio-temporal interactions [32].
Let $Y_{i j k}$ be the observed number of adverse pregnancy outcomes, where $i$ represents a given sub-county (1-290), $\boldsymbol{j}$ represents different periods (2017-2019) and $\boldsymbol{k}$ represents the different adverse pregnancy outcomes (1 - Low birthweight, 2 - Pre-term births, 3 -Stillbirths, 4 Neonatal deaths).

Let $\boldsymbol{n}_{i j k}$ be total live births for sub-county $\boldsymbol{i}(\boldsymbol{i}=\mathbf{1}, \ldots, 290)$ and time period $\boldsymbol{j}(\boldsymbol{j}=\mathbf{1}, \ldots, \mathbf{1 6})$ across all the adverse outcomes $\boldsymbol{k}(\boldsymbol{k}=\mathbf{1}, \mathbf{2}, \mathbf{3}, \mathbf{4})$.Due to the low rate of adverse pregnancy outcomes when compared to live births, and without the loss of generality, a binomial model for observed counts with a logit link function was used in the analysis.

i.e. We assume that the observed number of cases $Y_{i j k}$ arises from a binomial distribution, that is;

$Y_{i j k} \sim \operatorname{binomial}\left(n_{i j k}, \pi_{i j k}\right) i=1, \ldots, 290 ; j=2016, \ldots, 2019 ; k=1,2,3,4$

Here, $\pi_{i j k}$ represents the true, unknown risk in subcounty $\boldsymbol{i}$. time $\boldsymbol{j}$ and disease $\boldsymbol{k}$. Then the proportionate rate is specified on the logit scale as:

$$
\operatorname{Logit}\left(\boldsymbol{\pi}_{\boldsymbol{i j k}}\right)=\alpha_{k}+\boldsymbol{X}_{\boldsymbol{i}} \beta_{k}+\boldsymbol{X}_{\boldsymbol{i j}} \beta_{k}+\boldsymbol{\mu}_{\boldsymbol{i j k}}
$$

The parameter $\alpha_{k}$ is the baseline risk for a given adverse pregnancy outcome, $X_{i}$ and $X_{i j}$ are the time invariant spatial covariates and space-time varying covariates (ANC4 visits, maternal anaemia, LLINs, IPT2, Iron, Folate)

Table 1 Definition of adverse pregnancy indicators

\begin{tabular}{|c|c|c|c|}
\hline Data & Description & Spatial resolution & $\begin{array}{l}\text { Temporal } \\
\text { resolution }\end{array}$ \\
\hline Pre-term birth & $\begin{array}{l}\text { The spontaneous or the induced live delivery of a baby before } 37 \text { completed } \\
\text { gestational weeks [27]. }\end{array}$ & Sub-county & Yearly \\
\hline Low birth weight & $\begin{array}{l}\text { The birth weight of less than } 2500 \mathrm{~g} \text { (up to and including } 2499 \mathrm{~g} \text { ) and is consid- } \\
\text { ered one the principal measures of birth outcomes. }\end{array}$ & Sub-county & Yearly \\
\hline Stillbirth & $\begin{array}{l}\text { The intrauterine death of a fetus at any time during pregnancy/ls a baby born } \\
\text { with no signs of life at or after } 28 \text { weeks of gestation. Stillbirth rate, is consider a } \\
\text { proxy indicator of quality of care during labour and delivery [19]. }\end{array}$ & Sub-county & Yearly \\
\hline Neonatal deaths & $\begin{array}{l}\text { The death of a live born infant, irrespective of gestational age at birth, within } \\
\text { the first } 28 \text { completed days of life. }\end{array}$ & Sub-county & Yearly \\
\hline Live birth & $\begin{array}{l}\text { The complete expulsion/extraction from its mother a of a product of concep- } \\
\text { tion, irrespective of the duration of the pregnancy, which, after such separation, } \\
\text { breathes or shows any other evidence of life. }\end{array}$ & Sub-county & Yearly \\
\hline ANC -4 visits & $\begin{array}{l}\text { The number of women with a live birth in a given time period that received } \\
\text { antenatal care four or more times and is an indicator of access and use of the } \\
\text { health care facility during pregnancy. }\end{array}$ & Sub-county & Yearly \\
\hline Maternal anaemia & Is the haemoglobin concentration below $11 \mathrm{~g}$ per decilitre (g/dL) [28]. & Sub-county & Yearly \\
\hline Long-lasting insecticidal net (LLINs) & $\begin{array}{l}\text { This is a core vector control intervention with an anticipated lifespan of } 3 \text { years } \\
\text { and } 20 \text { washes based on its physical and chemical durability, that has been } \\
\text { shown to reduce malaria illness and mortality in endemic regions [29]. }\end{array}$ & Sub-county & Yearly \\
\hline $\begin{array}{l}\text { Intermittent preventive treatment } \\
-2 \text { doses (IPT2) }\end{array}$ & $\begin{array}{l}\text { This a curative dose of an effective antimalarial drug (e.g. sulfadoxine-pyrimeth- } \\
\text { amine) to all pregnant women during their routines antenatal care visit. }\end{array}$ & Sub-county & Yearly \\
\hline $\begin{array}{l}\text { Iron and } \\
\text { Folic Acid Supplementation }\end{array}$ & $\begin{array}{l}\text { Are essential micronutrient interventions used to reduce maternal anaemia, risk } \\
\text { of low birth weight, neural tube defects in pregnancy and improve the overall } \\
\text { pregnancy outcomes. }\end{array}$ & Sub-county & Yearly \\
\hline
\end{tabular}


respectively. Whereas $\beta_{k}$ are the vectors of regression coefficients for each pregnancy outcome that accounts for the varied risk gradients of the shared spatial and temporal components. i.e. they represent the relative weight of the contribution of the shared terms to the risk APOs. The spatio-temporal structure is defined by $\boldsymbol{\mu}_{\boldsymbol{i j} \boldsymbol{k}}$, which accounts for the possible variations of risk in the logit scale.

The joint spatial-temporal structure for each outcome was specified as follows

$$
\mu_{i j k}=\gamma_{k}^{s} u_{i}^{s}+u_{i k}^{s}+\gamma_{k}^{t} u_{j}^{t}+u_{j k}^{t}+v_{i j}
$$

With

$$
\sum_{k=1}^{4} \gamma_{k}^{s}=0 \text { and } \sum_{k=1}^{4} \log \gamma_{k}^{t}=0
$$

In this formulation $u_{i}^{s}$ are a set of common random effects associated with space, i.e. conditional autoregressive (CAR), $u_{i k}^{s}$ are outcome-specific random effects associated with space (CAR), $k=1,2,3,4, u_{j}^{t}$ are a set of common random effects associated with time, i.e. random walk of order 1 (RW1), $u_{j k}^{t}$ are outcome-specific random effects associated with time (RW1) [33-35]. The spacetime interaction term/heterogeneity of order two is represented by $v_{i j}$ i.e. it represents the possible variations not explained by the spatial and temporal effects in the model. A detailed description of the analyses is found in supplementary file 1 .

\section{Computation}

A two-chain Markov Chain Monte Carlo Simulation (MCMC) with 80,000 iterations and a burn-in of 4000 samples was used for estimating the model parameters. Both models were implemented in WinBUGS Version 1.4.3 (available at http://www.mrc-bsu.cam.ac.uk/bugs/ welcome.shtml). ArcMap 10.7.1 was used for creating risk maps (ESRI Inc., Redlands, CA, USA).

\section{Model diagnostics}

A random sample comprising $20 \%$ of the observed data points was drawn from the space-time cube to assess the overall model adequacy. The data with the removed points were then re-inputted into WinBUGS. The posterior estimates were then compared with the observed values. The posterior estimates (fixed effects, scaling parameters, and standard deviations of all random effects) were also assessed to check if the MCMC simulation had converged. i.e. A MC error/SD of less than $5 \%$ pointed to the model stability. Convergence of the MCMC chain was assessed visually by monitoring the trace plots for each parameter and analytically by the Gelman-Rubin statistics [36] (Supplementary file 1).

This analysis adheres to the Guidelines for Accurate and Transparent Health Estimates Reporting (GATHER) [37].

\section{Results}

Temporal trends in the burden of adverse pregnancy outcomes in Kenya from 2016 to 2019

A total of $2,896,659$ (95\% Uncertainty Interval: $2,811,500-2,981,817$ ) live births were reported in Kenya from January 2016 to December 2019. Overall, the total number of APOs increased by $14.2 \%$ (95\% UI: 14.0-14.5) from 88,816 cases in 2016 to 101,455 cases in 2019. In particular, low birth weight increased by $19.8 \%$ (95\% UI: 19.4-20.2) from 38,440 cases in 2016 to 46,051 cases in 2019 as shown in Table 2. The number of stillbirths increased by $5.5 \%$ (95\% UI: 5.1-5.8) from 16,888 cases in 2016 to 17,810 cases in 2019. Pre-term births increased by $16.3 \%$ (95\% UI: $15.8-16.8$ ) from 23,597 cases in 2016 to 27,437 cases in 2019. Neonatal deaths were the least reported events and the counts slightly increased by $2.7 \%$ (95\% UI: 2.4-3.0) from 9891 cases in 2016 to 10,157 cases in 2019 (Fig. 2).

\section{Low birth weight}

The low birth rate was estimated to be 4.5 (95\% UI: 4.4-4.7) per 100 live births, with the proportionate risk of low birth weight increasing from 4.3 (4.0-4.7) per 100 live births in 2016 to $4.8(4.5-5.1)$ per 100 live births in 2019 . Our estimates suggest that $60 \%$ or $173 / 290$ sub-counties exhibited a decline in the risk of low birth weight (ranging from an absolute difference of $7.5 \%$ in Mosop to $0.5 \%$ in Wajir South). However, the risk increased in $40 \%$ or $117 / 290$ sub-counties (from $15.7 \%$ in Kipkelion West to $1.8 \%$ in Navakholo). Malaria endemic sub-counties along the Indian Ocean coastline exhibited the highest risk over the study period, with the highest risk of 7.8 (95\% UI: 6.0-9.7) per 100 live births observed in 2018 (Supplementary

Table 2 Temporal changes in the burden of adverse pregnancy outcomes from 2016 to 2019. Among the adverse pregnancy outcomes considered in the study, the most and least common outcomes were low birth weight and neonatal deaths, respectively

\begin{tabular}{llllll}
\hline Year & $\mathbf{2 0 1 6}$ & $\mathbf{2 0 1 7}$ & $\mathbf{2 0 1 8}$ & $\mathbf{2 0 1 9}$ & Total \\
\hline Low Birth Weight & 38,440 & 23,162 & 43,150 & 46,051 & 150,803 \\
Stillbirths & 16,888 & 11,381 & 17,781 & 17,810 & 63,860 \\
Pre-term births & 23,597 & 15,672 & 24,976 & 27,437 & 91,682 \\
Neonatal deaths & 9891 & 5688 & 9959 & 10,157 & 35,695 \\
\hline
\end{tabular}




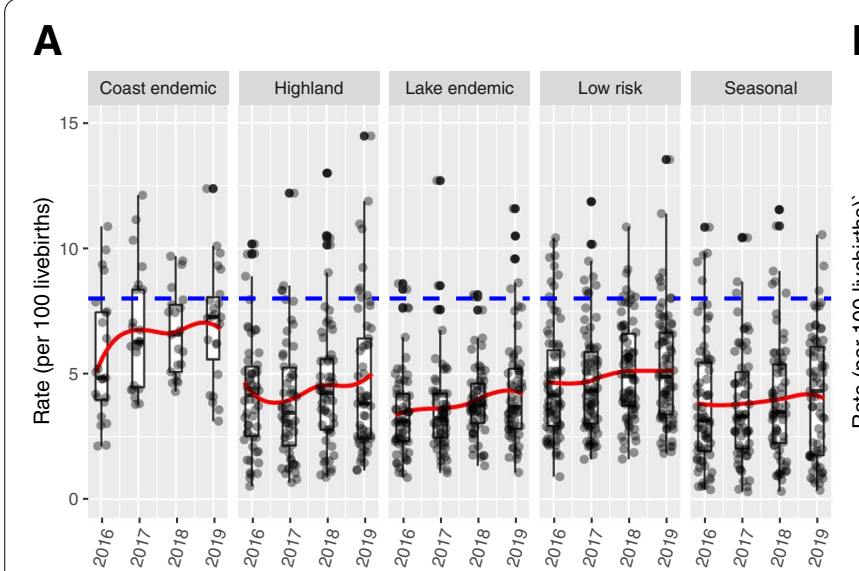

B

C

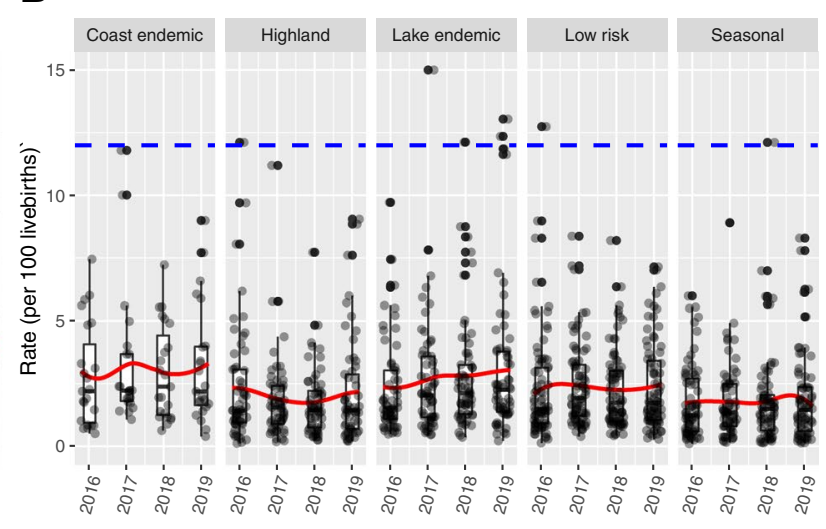

D
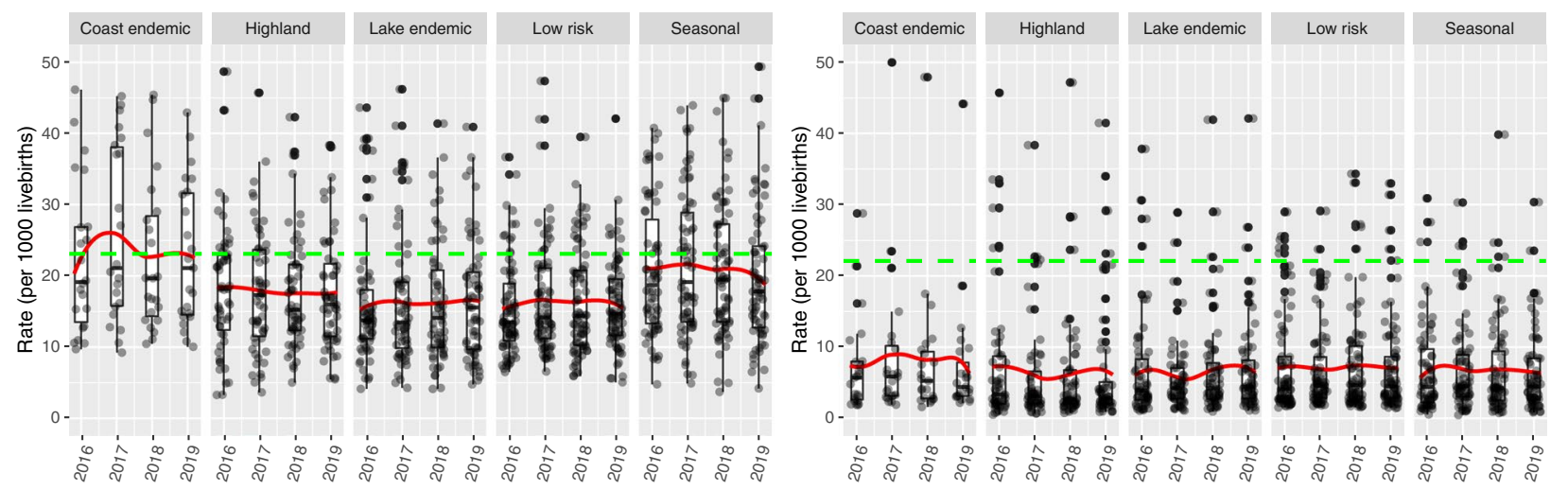

Fig. 2 Posterior estimates (mean) of adverse pregnancy outcomes stratified by malaria endemicity from January 2016 to December 2019 . A: Low birth weight per 100 live births, The blue dotted line (11.5 per 100 live births) represents the estimated national rate of LBW [38]. B: Pre-term birth rate (per 100 live births), the blue dotted line (12 per 100 live births) represents the national rate [39], (C); Stillbirth rate (per 1000 live births), the green dotted line represents the estimated the national rate [8]. D: Neonatal death rate (per 1000 live births), the green dotted line represents the estimated national rate [40]

file 2). Conversely, arid and semi-arid sub-counties in northern and eastern parts such as Mandera North, Mandera West, Eldas, Tarbaj, Banissa, Wajir South and Laisamis, exhibited low risk over the study period, as is shown in Fig. 3.

\section{Pre-term births}

The estimated pre-term birth rate was 2.3 (95\% UI: 2.2-2.5) per 100 live births, with 134 of 290 sub-counties exhibiting a decline in the risk pre-term births (ranging from an absolute difference of $11.6 \%$ in Olkalou to $0.01 \%$ in Borabu), while the risk increased for 156 sub-counties (ranging from to $8.3 \%$ in Jomvu to $0.02 \%$ in Gichugu) as shown in Fig. 3. Compared to other sub-counties, elevated risk was observed in a few sub-counties along the Indian Ocean coastline, whereas low risk was observed in arid and semi-arid sub-counties in the northern and eastern parts of Kenya. In 2019, elevated risk was observed in western
Kenya in Lurambi, Suna East, Kisumu Central and Matayos sub-counties.

\section{Stillbirths}

The median stillbirth rate in this study was estimated to be 18.7 (95\% UI: 18.0-19.4) per 1000 live births. Between 2016 to $2019,50 \%$ or $146 / 290$ sub-counties exhibited a decline in the risk stillbirths (ranging from an absolute difference of $20 \%$ in Kapsaret, to $0.003 \%$ in Ndhiwa), while the risk of stillbirths increased in 144 sub-counties (ranging from to $5.7 \%$ in Narok North to $0.01 \%$ in Kabondo Kasipul). Elevated risk was observed in malaria-endemic sub-counties located along the Indian ocean coastline followed by seasonal endemic subcounties (Supplementary file 2). In 2019, the risk ranged from 87.9 (95\% UI: 80.2-96.3) per 1000 live births to 4.1 (95\% UI: 2.5-6.5) per 1000 live births with elevated risk observed in Narok North and Narok South sub-counties (Fig. 4). 

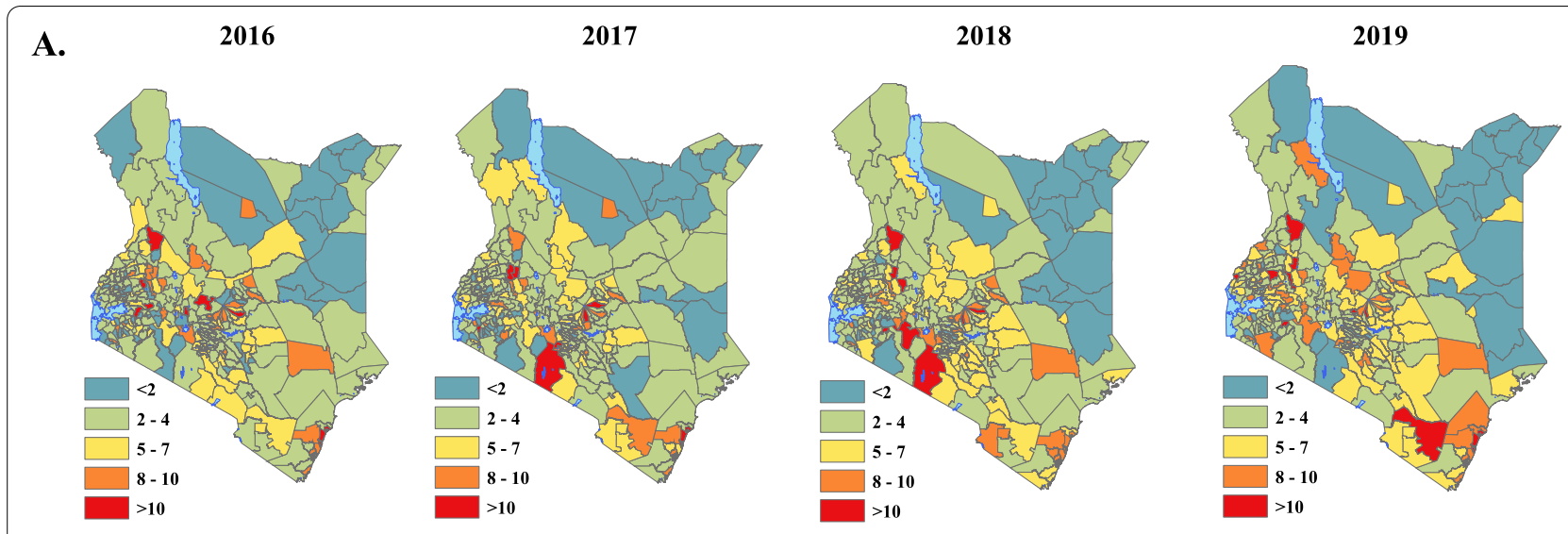

B.

2016

2017

2018

2019
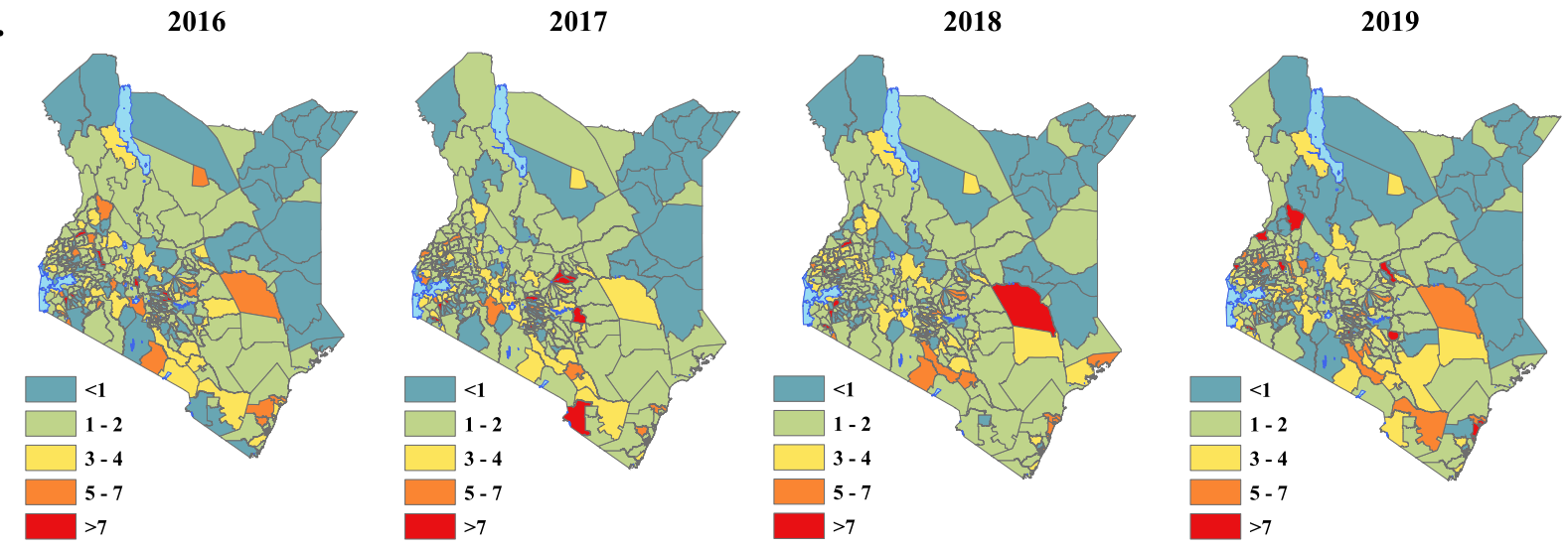

\begin{tabular}{cccccc}
0 & 200 & 400 & 800 & 1,200 & 1,600 \\
\hline & $\square$ & $\square$ & & & KM
\end{tabular}



Fig. 3 Maps showing the changing spatial evolution of the posterior median risk of (A) Low-birth weight per 100 live births, B Pre-term births per 100 live births

\section{Neonatal deaths}

The neonatal death rate was estimated to be 6.9 (6.4-7.4) per 1000 live births, with the rate declining by $2.8 \%$, from 7.1 per 1000 live births in 2016 to 6.9 per 1000 live births in 2019. Over this period, $48 \%$ or $139 / 290$ sub-counties exhibited a decline in the risk, with the fastest decline rate $(-2.6 \%)$ being observed in Kitutu Chache South sub-county. The risk also increased in $52 \%$ or $151 / 290$ sub-counties, with Mvita sub-county recording the highest rise of $1.5 \%$. Elevated risk was observed in sub-counties with urban informal settings that are characterised by poverty and poor access to maternal health services, such as Kibra and Mvita sub-counties. Similarly, malaria-endemic subcounties along the Indian Ocean coastline and subcounties with seasonal transmission trends exhibited elevated risk (Fig. 4).

\section{Impact of covariates on the proportionate rate of adverse pregnancy outcomes}

The spatio-temporal distribution of covariates is shown in (Supplementary file 2). There was a positive association between maternal anaemia levels, the use of LLINs, iron supplements, folate supplements on the proportionate rate of adverse pregnancy outcomes. Conversely, our results suggested a significant negative association between adverse pregnancy outcomes and the use of IPT2 over the study period. Antenatal care was also negatively associated with adverse pregnancy outcomes, though this association was not statistically significant (Table 3).

The shared risk patterns of adverse pregnancy outcomes varied sub-nationally as displayed in Fig. 5. Shared high risk was concentrated in sub-counties located along the Indian Ocean coastline, central regions and the 

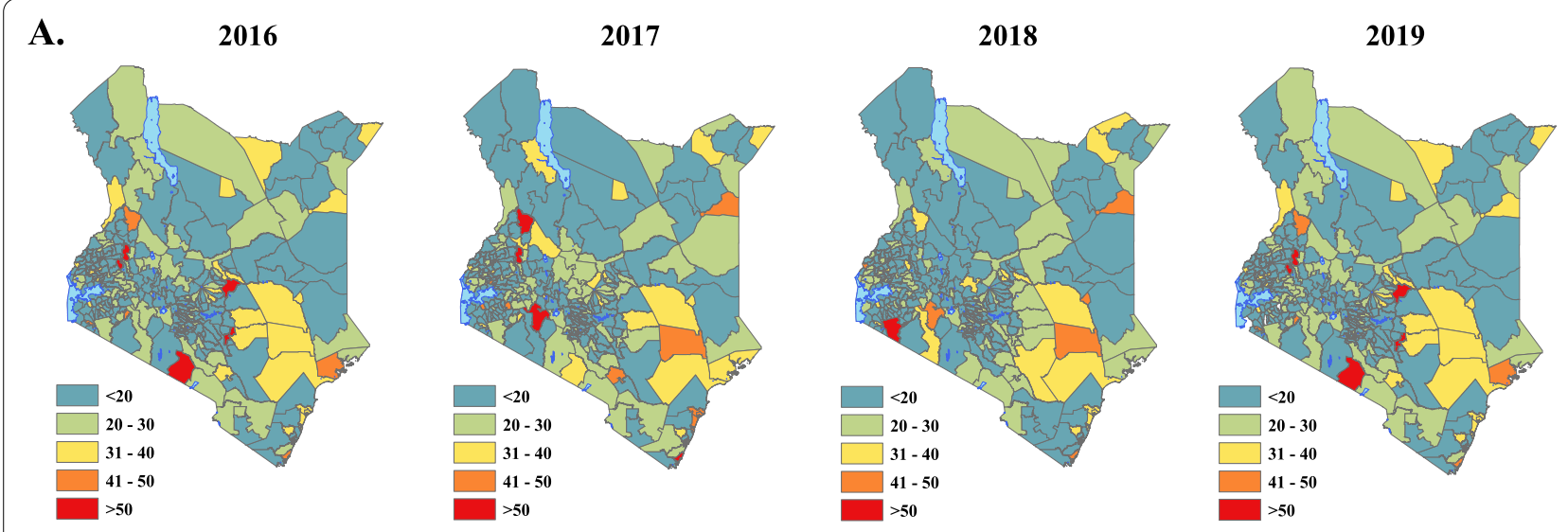

B.

2016



2017

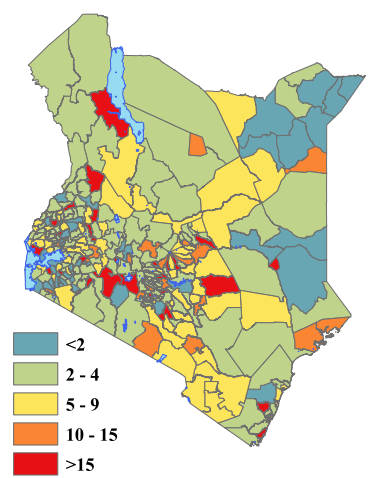

2018

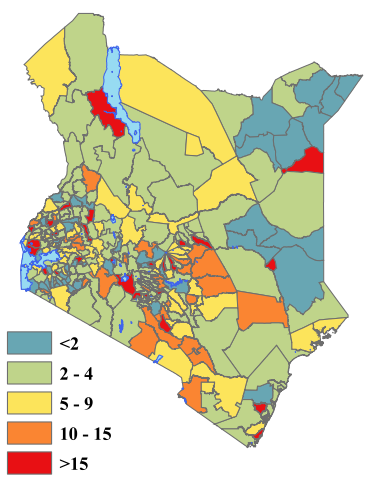

2019



$\stackrel{N}{N}$

Fig. 4 Maps showing the changing spatial evolution of the posterior median risk of (A) Stillbirths per 1000 live births, (B) Neonatal deaths per 1000 live births

Table 3 Posterior estimates of covariates

\begin{tabular}{|c|c|c|}
\hline Covariate & Posterior estimate & $\begin{array}{l}95 \% \text { Uncertainty } \\
\text { Interval }\end{array}$ \\
\hline Antenatal care -4 visits & -0.035 & $-0.105,0.100$ \\
\hline Maternal anaemia & 0.059 & $-0.006,0.293$ \\
\hline $\begin{array}{l}\text { Long-lasting insecticidal- } \\
\text { treated nets (LLINs) }\end{array}$ & 0.016 & $-0.018,0.073$ \\
\hline $\begin{array}{l}\text { Intermittent preventive } \\
\text { treatment }-2 \text { doses } \\
\text { (IPT2) }\end{array}$ & -0.071 & $-0.208,-0.023$ \\
\hline Iron & 0.034 & $-0.012,0.145$ \\
\hline Folate & 0.084 & $-0.020,0.356$ \\
\hline
\end{tabular}

western parts of Kenya. These sub-counties were Nyali, Rongo, Kilifi North and Nakuru East. Conversely, the low shared risk was concentrated mainly in sub-counties located in Kenya's northern and eastern parts such as Tarbaj, Mandera North/West, Eldas and Wajir North.

\section{Discussion}

We employed a Bayesian hierarchical binomial model that simultaneously analysed the spatial pattern and temporal trend of adverse pregnancy outcomes (APOs) in Kenya between 2016 and 2019. Our modelling framework allowed us to incorporate information from multiple pregnancy outcomes that shared risk factors over the spatial extents and time periods. Information across the outcomes was specified via the spatial and temporal correlation structures, and our model was adjusted for both the covariate effect and an unstructured space-time interaction effect. To our knowledge, this analysis represents the first sub-national, high-resolution estimation of adverse pregnancy outcomes concomitantly over space and time in Kenya, which is a crucial input for strengthening national and county efforts towards the realisation of Universal Health Coverage (UHC) and achieving global health targets. Estimates of maternal health outcomes have historically relied on periodic household 


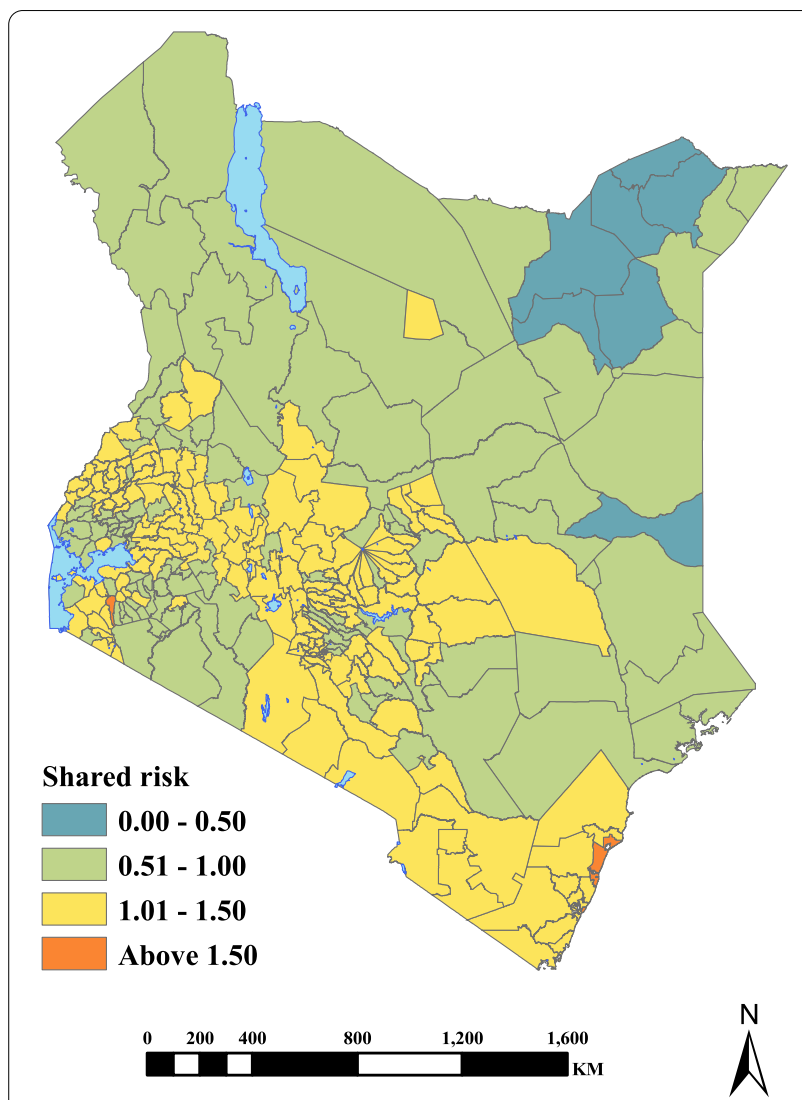

Fig. 5 Estimated posterior median for the shared components in the model, that captures the underlying risk common to all adverse outcomes

surveys such as the DHS and MIS. However, their sampling design limits their scope as they are best suited to yield estimates at the national or regional level. Compared to the unstable national-level estimates, our estimates provide greater descriptive granularity and give a baseline for bolstering maternal health programs in highrisk sub-counties.

The risk of preventable adverse pregnancy outcomes increased between 2016 and 2019 and was non-uniform across all the outcomes. Low birth weight (LBW) is considered a composite measure of foetal growth and an underlying determinant/contributor of adverse outcomes, and has been associated with an increased risk of poor physical and cognitive development in infants $[41,42]$. Our results suggest an elevated risk of LBW in sub-counties located along the Indian ocean coastline which may signal the role of malaria in pregnancy. On the other hand, the low risk observed in resource-constrained sub-counties located in the north-eastern parts of Kenya may signal limited/unreliable data leading to an underestimation of its burden. This heterogeneous burden of LBW calls into question the implementation of national policies particularly in resource constrained sub-counties and urban informal settlements. Results suggest the need for more targeted approaches; particularly in sub-counties with urban informal settings where LBW contributes to neonatal deaths but remain underestimated [43, 44].

The overall pre-term birth rate was markedly lower than 9 per 100 live births previously estimated [45, 46]. By 2019, the pre-term birth rate ranged between 0.12 to 13.0 per 100 live births, with elevated estimates being observed in a few sub-counties located in the south-western parts of Kenya and along the Indian ocean coastline. However, low rates were consistently observed in the north-eastern parts of Kenya which may be attributed to limited data quantities over the period. Thus, to better understand the epidemiology of pre-term birth in low-risk sub-counties the volume and quality of routinely collected data need to be strengthened. Conversely, the elevated burden observed in some sub-counties in the south-western parts, may be reflective of the unresolved burden of pre-term births in unintended adolescent pregnancies [42, 47].

Our stillbirth estimate of 18.7 per 1000 live births was significantly lower than previous estimates for Kenya and SSA $[8,46]$. The rate declined by $6.7 \%$, from 19.4 per 1000 live births in 2016 to 18.1 per 1000 live births in 2019. The slow decline of stillbirths primarily attributed to intrapartum deaths [8], may be reflective on the inadequate global and national attention in mitigating the burden [11]. Additionally, generated estimates need to be interpreted cautiously due to under-reporting attributed to recall bias, inconsistent definitions/misclassification of fresh versus macerated stillbirths and omission. This may limit comparison with other international studies using diverse classification criteria $[7,11]$. Improving the quality of care and birth and registry data will likely contribute to accurate estimates sub-nationally.

Neonatal death rate is an important index for tracking progress towards the attainment of SDG targets, that seeks to reduce neonatal mortality to at least 12 deaths per 1000 live births. Understanding its spatial extents and temporal trends is essential/critical due to its rising share in under-five mortality in Kenya [25]. Our estimates was markedly lower compared to a facility-based study that estimated the neonatal death rate to be 16 per 1000 live births [46]. Huge disparities in neonatal deaths persisted across sub-counties, with elevated risk observed in malaria-endemic regions located along the Indian Ocean coastline and Lake Victoria region. The highest risk was consistently observed in Kibra sub-county, with approximately 75 deaths in 1000 live births [48]. It is important to note that Kibra inhabits the largest urban informal settlement in Africa [44]. Thus, the elevated risk may have been compounded by poor sanitation practices and 
over-stretched facilities in the densely populated informal settlements [49].

Our results suggest that disparities in the spatial extents of APOs may have been driven by demand-side barriers and supply-side challenges, with low-resource counties disproportionately vulnerable to adverse outcomes. Recent studies highlight a heterogenous timing and coverage of ANC4 across sub-counties in Kenya, with poorer/rural sub-counties in northern Kenya exhibiting low coverage levels [50, 51]. The current guidance from the WHO suggesting at least four comprehensive and targeted visits $[16,52]$. However, a confidential inquiry into maternal deaths in Kenya indicated that only one in five women attending ANC had at least 4 ANC visits in 2014 [53]. ANC attendance and coverage are essential as they are positively associated with increased use of birth attendants, reducing the risk of adverse pregnancy outcomes. Pregnant women are screened for anaemia, HIV, syphilis, urinalysis, and blood typing (ANC profile) during these visits. Additionally, they receive vital micronutrient supplementation essential to both the mother and her unborn child among other interventions such as educational information on safe motherhood. However, regional disparities may signify the inequitable access and the widespread unmet need of ANC services particularly in arid, resource-constrained, rural and pastoralist dominated sub-counties located eastern and northern parts of Kenya as previously documented [11, 54-56].

Increasing concerns about the shortage and distribution of skilled healthcare workers sub nationally, adequacy of pre-existing healthcare infrastructure, stakeholder non-involvement in policy formulation has previously been raised $[10,57]$. These concerns have been exacerbated by the distance to health facility, timely access to quality maternal care, cost of transport, poverty, illiteracy, religious and social-cultural beliefs and tendencies. These factors tend to suppress the uptake of facility-based delivery with only the complicated cases being referred to the facility [50, 51, 58-61]. This is more pronounced in the rural and pastoralist communities that prefer traditional birth attendants $[62,63]$. According to the Kenya Health Workforce Status Report in 2017, the nurse-to-population ratio of $0.1 / 10,000$ in arid and semi-arid sub-counties in northern Kenya was markedly lower than 9.7/10,000 in subcounties Nairobi [64]. This might have contributed by high attrition rates of health staff in these sub-counties due to poor infrastructure and insecurity. This has led to poor utilisation of public health facilities, leading to increased home deliveries [61]. Our results lend credence to efforts to strengthen the healthcare workforce through policy, planning, adequate financing, recruitment, training and deployment. This may help to improve sub-county health systems and help achieve global and national health targets such as the universal coverage of skilled birth attendants [65].

In low-resource sub-counties in northern Kenya, macronutrient/maternal undernutrition - caused by inadequate intake of essential minerals and vitamins; contributes to anaemia in pregnancy, which might also precipitate APOs [66]. Additionally, infectious diseases such as malaria and human immunodeficiency virus (HIV) have previously been associated with an increased risk of pre-term deliveries, stillbirths, and neonatal deaths. The burden is more pronounced in sub-counties located in western Kenya [67-73]. Sexually transmitted infections such as chlamydia, gonorrhoea, trichomoniasis and syphilis have also been associated with an increased risk of stillbirths and low birth weight among pregnant women in rural settings [74-76].

\section{Implication of study}

Despite the achievements in maternal and child healthrelated programs over the recent decades, preventable adverse pregnancy outcomes remain a key challenge to pregnant women and their infants. The study presents a unique dimension for prioritising high-risk areas and increasing the efficiency of control programmes to alleviate the burden imposed by preventable APOs. This will accelerate the attainment of maternal health targets and align sub-county estimates with global and national priorities. Crucially, our estimates can help to raise awareness of growing inequities in Kenya's diverse and growing population inhabiting different regions with diverse levels of social and economic development.

The shared risk estimates underscore the need to establish integrated suites of interventions (policy/care package/services) at meaningful policy levels to reduce missed opportunities/comprehensively address healthcare needs of pregnant women at the sub-county level, especially through nutrition-specific and nutrition-sensitive interventions. Compared to the national level estimates, our joint estimates resonate with Kenya's broader maternal health objectives, focusing on disease patterns and monitoring/accelerating progress in maternal health care equity as outlined in the SDGs. Our findings also inform the need of mapping the overlapping burden of infectious diseases and APOs in Kenya. Study approaches can also be applied to other health outcomes with shared risk factors.

As more data on maternal health outcomes becomes available, underestimation stemming from inaccurate reporting/documentation can be used to flag subcounties with less reliable data on other maternal health 
indicators and help channel investments to sub-counties with poor surveillance systems, constrained diagnostic capacity and unmet reproductive health needs. Overall, the local burden of disease can be used to prioritise appropriate, cost-effective interventions targeting the poor, rural sub-counties and informal settlements in urban areas will likely address the wide disparities and accelerate progress in maternal and newborn health. There is also a pressing need to understand more about APO's risk to urban slum dwellers in Kenya who are disproportionately bombarded with chronic illness, maternal illiteracy, and socio-economic instability.

\section{Study strengths and limitations}

At a much higher level of spatio-temporal precision, we demonstrate the importance of a functional health information system in providing routine estimates between periodic and expensive survey estimates. Facility-based data can help inform the local burden of disease. The continual adoption of standard definitions and consistent measurement procedures will be critical for meaningful comparisons towards the attainments of SDGs. The Bayesian shared component model adds versatility to substantive epidemiological questions. Its ability to borrow strength from outcomes and across neighbouring sub-counties makes it an important tool for monitoring the evolution of facility-based maternal health disparities across the continuum of care.

Our findings need to be interpreted cautiously due to several methodological challenges that might have confounded our estimates. First, the study presents facility-based estimates that may not be generalisable to the entire population, especially for women delivering outside the public health facilities. This may be due to the geographic underrepresentation of public antenatal clinics. Secondly, we cannot discount the potential incompleteness of our data, disproportionately available in malaria endemic sub-counties with more robust surveillance infrastructure and higher uptake of antenatal care services. Third, misclassification of pregnancy outcomes; e.g. neonatal deaths as stillbirths, pre-term birth as low birth weight (or vice versa), might have led to underestimating some adverse outcomes. This may in part explain previous concerns raised on the use of routine health metrics for international comparisons [77]. Fourth, although we included covariates to stabilise our estimates, our study was ecological with data aggregated at the subcounty and yearly level; this might have led to residual confounding. Fifth, our study did not account for the adverse outcomes during the different pregnancy semesters, pregnancy history, underlying conditions and parity which has previously been associated with adverse outcomes.

\section{Conclusions}

Our study primarily modelled the geographical distribution and risk of adverse pregnancy outcomes among women presenting at public health facilities in Kenya. High-resolution estimates via the Bayesian joint spatio-temporal models were used to strengthen inference by borrowing information across adjacent regions and adverse pregnancy outcomes with common risk factors. As facility-based deliveries continue to increase, there is an urgent need to improve the quality and volume of data, including standardising definitions, measurements and reporting. This will enable the implementation of appropriate context-specific interventions and help reduce associated APOs. Over the coming decades, understanding the joint burden of overlooked maternal health disparities indexed in space and time will enable the implementation of health measures with increasing granularity, simultaneously decreasing the risk of adverse pregnancy outcomes.

\section{Abbreviations \\ CAR: Conditional autoregressive; DIC: Deviance information criterion; MCMC: Markov chain Monte Carlo; DHIS: District health information system; SSA: Sub-Saharan Africa; SDG: Sustainable development goal; WHO: World health organization; ENAP: Every newborn action plan; EPMM: Ending preventable maternal mortality.}

\section{Supplementary Information}

The online version contains supplementary material available at https://doi. org/10.1186/s12889-021-12210-9.

Additional file 1: Supplementary file 1: Additional modelling details

Additional file 2: Supplementary file 2: Additional data descriptions, methodological information and results

Acknowledgements

We acknowledge the support of the Kenya Ministry of Health for providing access to the DHIS2 database. This research was supported and funded by the University of KwaZulu Natal, College of Health Sciences.

\section{Authors' contributions}

JNO and BS participated in conceptualising the study, developed the statistical model and analysed the model results. JNO wrote the manuscript and $\mathrm{BS}$ revised it critically. All authors approved the final version of the manuscript.

\section{Funding}

This study has been supported by University of KwaZulu-Natal, College of Health Sciences research scholarship scheme. The funder of the study had no role in study design, data collection, data analysis, data interpretation, or writing of the report

\section{Availability of data and materials}

The datasets analysed during the current study are available in the Kenya District Health Information Software online database (DHIS2) repository, http:// www.hiskenya.org. The shapefile used in the study was downloaded from https://data.humdata.org/dataset/ken-administrative-boundaries. 


\section{Declarations}

\section{Ethics approval and consent to participate}

Ethical approval was received from both the University of KwaZulu- Natal, reference number: BE290/18 and the Kenya Medical Research Institute (KEMRI) Review Board, reference number: KEMRI/RES/7/3/1. The study used secondary data only, all publicly available to registered users from online data repositories. No individual patient-level data was used in this publication

\section{Consent for publication}

Not applicable.

\section{Competing interests}

The authors declare that they have no competing interests

\section{Author details}

${ }^{1}$ Discipline of Public Health Medicine, College of Health Sciences, University of KwaZulu-Natal, 2nd Floor George Campbell Building, Howard College Campus, Durban 4001, South Africa. ${ }^{2}$ Department of Management Science and Technology, The Technical University of Kenya, Nairobi, Kenya. ${ }^{3}$ Ignite Lab, Global Research Institute, William and Mary, Williamsburg, Virginia, USA. ${ }^{4}$ Centre for Tropical Medicine and Global Health, Nuffield Department of Medicine, University of Oxford, Oxford, UK. ${ }^{5}$ Department of Health Metrics Sciences, University of Washington, Seattle, USA.

Received: 28 October 2020 Accepted: 10 November 2021

Published online: 30 December 2021

\section{References}

1. UNICEF: Trends in maternal mortality 2000 To 2017: estimates by WHO, UNICEF, UNFPA, World Bank Group and the United Nations Population Division. 2019.

2. United Nations. Progress on the sustainable development goals, the gender snapshot 2019. New York: UN Women; 2019.

3. World Health Organization: Trends in maternal mortality 2000 to 2017 : estimates by WHO, UNICEF, UNFPA, World Bank Group and the United Nations Population Division. 2019

4. Lean SC, Derricott H, Jones RL, Heazell AE. Advanced maternal age and adverse pregnancy outcomes: a systematic review and meta-analysis. PLoS One. 2017;12(10):e0186287.

5. World Health Organization: Quality, equity, dignity: the network to improve quality of care for maternal, newborn and child health: strategic objectives. 2018.

6. Alkema L, Chou D, Hogan D, Zhang S, Moller A-B, Gemmill A, et al. Global, regional, and national levels and trends in maternal mortality between 1990 and 2015, with scenario-based projections to 2030: a systematic analysis by the UN maternal mortality estimation inter-agency group. Lancet. 2016:387(10017):462-74.

7. Wang H, Abajobir AA, Abate KH, Abbafati C, Abbas KM, Abd-Allah F, et al. Global, regional, and national under-5 mortality, adult mortality, age-specific mortality, and life expectancy, 1970-2016: a systematic analysis for the global burden of disease study 2016. Lancet. 2017;390(10100):1084-150.

8. Lawn JE, Blencowe H, Waiswa P, Amouzou A, Mathers C, Hogan D, et al. Stillbirths: rates, risk factors, and acceleration towards 2030. Lancet. 2016;387(10018):587-603

9. United Nations inter-agency Group for Child Mortality Estimation: global, regional, and national levels and trends in under- 5 mortality between 1990 and 2015, with scenario-based projections to 2030: a systematic analysis by the UN inter-agency Group for Child Mortality Estimation. In: Lancet. 2019:386:2275-86.

10. Njuguna J, Kamau N, Muruka C. Impact of free delivery policy on utilization of maternal health services in county referral hospitals in Kenya. BMC Health Serv Res. 2017;17(1):429.

11. Keats EC, Ngugi A, Macharia W, Akseer N, Khaemba EN, Bhatti Z, et al. Progress and priorities for reproductive, maternal, newborn, and child health in Kenya: a countdown to 2015 country case study. Lancet Glob Health. 2017:5(8):e782-95.
12. World Health Organization: Strategies towards ending preventable maternal mortality (EPMM). 2015.

13. World Health Organization: WHO technical consultation on newborn health indicators: every newborn action plan metrics, Ferney Voltaire, France, 3-5 December 2014. In.: World Health Organization; 2015.

14. World Health Organization: Global nutrition targets 2025: policy brief series. In.: World Health Organization; 2014.

15. Keats EC, Macharia W, Singh NS, Akseer N, Ravishankar N, Ngugi AK, et al. Accelerating Kenya's progress to 2030: understanding the determinants of under-five mortality from 1990 to 2015. BMJ Glob Health. 2018;3(3):e000655.

16. Ministry of Health: Kenya health policy 2014-2030: towards attaining the highest standard of health. In. Nairobi; 2014.

17. Seabrook JA, Smith A, Clark AF, Gilliland JA. Geospatial analyses of adverse birth outcomes in southwestern Ontario: examining the impact of environmental factors. Environ Res. 2019;172:18-26.

18. Campbell EE, Gilliland J, Dworatzek PD, De Vrijer B, Penava D, Seabrook JA Socioeconomic status and adverse birth outcomes: a population-based Canadian sample. J Biosoc Sci. 2018;50(1):102-13.

19. Neogi SB, Sharma J, Negandhi P, Chauhan M, Reddy S, Sethy G. Risk factors for stillbirths: how much can a responsive health system prevent? BMC Pregnancy Childbirth. 2018;18(1):33.

20. Miao H, Li B, Li W, Yao F, Chen Y, Chen R, et al. Adverse birth outcomes in Guangdong province, China, 2014-2017: A spatiotemporal analysis of 2.9 million births. BMJ Open. 2019;9(11):e030629.

21. Librero J, Ibañez B, Martínez-Lizaga N, Peiró S, Bernal-Delgado E. On behalf of the Spanish atlas of medical practice variation research $\mathrm{G}$ : applying spatio-temporal models to assess variations across health care areas and regions: lessons from the decentralized Spanish National Health System. PLoS One. 2017;12(2):e0170480.

22. National Coordinating Agency for Population and Development (NCAPD) [Kenya] MoH, Central Bureau of Statistics, ORC Macro,: Kenya Service Provision Assessment Survey, 2004. In.: Ministry of Health; 2005.

23. Ministry of Health: Kenya Health Sector Referral Strategy. In. Nairobi; 2014.

24. Kenya National Bureau of statistics: economic survey report. In.; 2020.

25. Kenya National Bureau of Statistics, Ministry of Health/Kenya, national AIDS control council/Kenya, Kenya medical research institute, population NCf, development/Kenya: Kenya demographic and health survey 2014. In. Rockville, MD, USA; 2015.

26. World Health Organization: United Nations Children's Fund. Every Newborn: An action plan to end preventable deaths [Internet]. Geneva; 2014. In.; 2017.

27. Soon BT. The global action report on preterm birth. Geneva: World Health Organization; 2012.

28. World health Organization: Haemoglobin concentrations for the diagnosis of anaemia and assessment of severity. In.; 2011.

29. Choi L, Pryce J, Richardson M, Lutje V, Walshe D, Garner P: Guidelines for malaria vector control. World Health Organization 2019:1-171.

30. Assoum M, Ortu G, Basáñez M-G, Lau C, Clements AC, Halton K, et al. Spatiotemporal distribution and population at risk of soil-transmitted helminth infections following an eight-year school-based deworming programme in Burundi, 2007-2014. Parasit Vectors. 2017;10(1):583.

31. Slater $\mathrm{H}$, Michael E. Mapping, Bayesian geostatistical analysis and spatial prediction of lymphatic filariasis prevalence in Africa. PLoS One. 2013;8(8):e71574

32. Downing A, Forman D, Gilthorpe MS, Edwards KL, Manda SO. Joint disease mapping using six cancers in the Yorkshire region of England. Int J Health Geogr. 2008;7(1):41.

33. Knorr-Held L. Bayesian modelling of inseparable space-time variation in disease risk. Stat Med. 2000;19(17-18):2555-67.

34. Richardson S, Abellan JJ, Best N. Bayesian spatio-temporal analysis of joint patterns of male and female lung cancer risks in Yorkshire (UK). Stat Methods Med Res. 2006;15(4):385-407.

35. Knorr-Held L, Best NG. A shared component model for detecting joint and selective clustering of two diseases. J R Stat Soc A (Stat Soc). 2001;164(1):73-85.

36. Gelman A, Rubin DB. Inference from iterative simulation using multiple sequences. Stat Sci. 1992;7(4):457-72.

37. Stevens GA, Alkema L, Black RE, Boerma JT, Collins GS, Ezzati M, et al. Guidelines for accurate and transparent health estimates reporting: the GATHER statement. PLoS Med. 2016;13(6):e1002056. 
38. World Health Organization: UNICEF-WHO low birthweight estimates: levels and trends 2000-2015. In.: World Health Organization; 2019.

39. Watkins K: The state of the World's children 2016: a fair chance for every child: ERIC; 2016.

40. World Health Organization: Trends in maternal mortality: 1990-2015: estimates from WHO, UNICEF, UNFPA, World Bank Group and the United Nations Population Division. In.: World Health Organization; 2015.

41. He Z, Bishwajit G, Yaya S, Cheng Z, Zou D, Zhou Y. Prevalence of low birth weight and its association with maternal body weight status in selected countries in Africa: a cross-sectional study. BMJ Open. 2018;8(8):e020410.

42. Blencowe H, Krasevec J, de Onis M, Black RE, An X, Stevens GA, et al. National, regional, and worldwide estimates of low birthweight in 2015, with trends from 2000: a systematic analysis. Lancet Glob Health. 2019;7(7):e849-60.

43. Nyamasege CK, Kimani-Murage EW, Wanjohi M, Kaindi DWM, Ma E, Fukushige $\mathrm{M}$, et al. Determinants of low birth weight in the context of maternal nutrition education in urban informal settlements, Kenya. J Dev Orig Health Dis. 2019;10(2):237-45.

44. Wanjohi MN, Ogada I, Wekesah FM, Khayeka-Wandabwa C, KimaniMurage EW: Relationship between maternal body composition during pregnancy and infant's birth weight in Nairobi informal settlements, Kenya. BMJ Nutrition, Prevention \& Health 2020:bmjnph-2019-000060.

45. World Health Organization: maternal and child epidemiology estimation group (MCEE), child causes of death, by country and by region, 2000-2016. 2018.

46. Waiswa P, Higgins BV, Mubiri P, Kirumbi L, Butrick E, Merai R, et al. And the preterm birth initiative $\mathrm{K}$, Uganda implementation research C: pregnancy outcomes in facility deliveries in Kenya and Uganda: a large crosssectional analysis of maternity registers illuminating opportunities for mortality prevention. PLoS One. 2020;15(6):e0233845.

47. Omoro T, Gray SC, Otieno G, Mbeda C, Phillips-Howard PA, Hayes T, et al. Teen pregnancy in rural western Kenya: a public health issue. Int J Adolesc Youth. 2018;23(4):399-408.

48. Kimani-Murage EW, Fotso J-C, Egondi T, Abuya B, Elungata P, Ziraba A et al. Trends in childhood mortality in Kenya: the urban advantage has seemingly been wiped out. Health Place. 2014;29:95-103.

49. Owiti A, Oyugi J, Essink D. Utilization of Kenya's free maternal health services among women living in Kibera slums: a cross-sectional study. Pan Afr Med J. 2018;30(1):86.

50. Wairoto KG, Joseph NK, Macharia PM, Okiro EA. Determinants of subnational disparities in antenatal care utilisation: a spatial analysis of demographic and health survey data in Kenya. BMC Health Serv Res. 2020;20(1):665.

51. Keats EC, Akseer N, Bhatti Z, Macharia W, Ngugi A, Rizvi A, et al. Assessment of inequalities in coverage of essential reproductive, maternal, newborn, child, and adolescent health interventions in Kenya. JAMA Netw Open. 2018;1(8):e185152.

52. Tunçalp Ö, Pena-Rosas JP, Lawrie T, Bucagu M, Oladapo OT, Portela A, et al. WHO recommendations on antenatal care for a positive pregnancy experience-going beyond survival. Bjog. 2017;124(6):860-2.

53. Ministry of Health: Saving mothers'lives 2017. Confidential Inquiry into Maternal Deaths in Kenya. In. Nairobi; 2017.

54. Newman Owiredu M, Newman L, Nzomo T, Conombo Kafando G, Sann $\mathrm{S}$, Shaffer N, et al. Elimination of mother-to-child transmission of HIV and syphilis: a dual approach in the African region to improve quality of antenatal care and integrated disease control. Int J Gynecol Obstet. 2015;130:S27-31.

55. Van Eijk AM, Bles HM, Odhiambo F, Ayisi JG, Blokland IE, Rosen DH, et al. Use of antenatal services and delivery care among women in rural western Kenya: a community based survey. Reprod Health. 2006;3(1):2.

56. Fleming E, Oremo J, O'Connor K, Odhiambo A, Ye T, Oswago S, et al. The impact of integration of rapid syphilis testing during routine antenatal services in rural Kenya. J Sex Transm Dis. 2013;2013:674584.

57. Lang'at E, Mwanri L. Healthcare service providers' and facility administrators' perspectives of the free maternal healthcare services policy in Malindi District, Kenya: a qualitative study. Reprod Health. 2015;12(1):59.

58. Ochako R, Fotso J-C, Ikamari L, Khasakhala A: Utilization of maternal health services among young women in Kenya: insights from the Kenya demographic and health survey, 2003. BMC Pregnancy Childbirth 2011, 11(1):1.

59. Magadi MA, Madise NJ, Rodrigues RN. Frequency and timing of antenatal care in Kenya: explaining the variations between women of different communities. Soc Sci Med. 2000:51(4):551-61.
60. Riang'a RM, Nangulu AK, JEW B. Perceived causes of adverse pregnancy outcomes and remedies adopted by Kalenjin women in rural Kenya. BMC Pregnancy Childbirth. 2018;18(1):408.

61. Moindi RO, Ngari MM, Nyambati VC, Mbakaya C. Why mothers still deliver at home: understanding factors associated with home deliveries and cultural practices in rural coastal Kenya, a cross-section study. BMC Public Health. 2015;16(1):114.

62. Ogolla JO: Factors associated with home delivery in west Pokot County of Kenya. Advances in public health 2015, 2015.

63. Kamau TW. The utilization of skilled delivery attendance from maternal shelters in Samburu County, north Rift Valley. Kenya: University of Nairobi; 2018.

64. Ministry of Health: Kenya Health Workforce Report,. In. Nairobi; 2017.

65. Maina I, Wanjala P, Soti D, Kipruto H, Droti B, Boerma T. Using healthfacility data to assess subnational coverage of maternal and child health indicators, Kenya. Bull World Health Organ. 2017;95(10):683.

66. Odhiambo JN, Sartorius B. Mapping of anaemia prevalence among pregnant women in Kenya (2016-2019). BMC Pregnancy Childbirth. 2020;20(1):1-11.

67. Xiao $P-L$, Zhou Y-B, Chen Y, Yang M-X, Song X-X, Shi Y, et al. Association between maternal HIV infection and low birth weight and prematurity: a meta-analysis of cohort studies. BMC Pregnancy Childbirth. 2015;15(1):246

68. Arab K, Spence AR, Czuzoj-Shulman N, Abenhaim HA. Pregnancy outcomes in HIV-positive women: a retrospective cohort study. Arch Gynecol Obstet. 2017;295(3):599-606.

69. Wedi COO, Kirtley S, Hopewell S, Corrigan R, Kennedy SH, Hemelaar J. Perinatal outcomes associated with maternal HIV infection: a systematic review and meta-analysis. Lancet HIV. 2016;3(1):e33-48.

70. Poespoprodjo JR, Fobia W, Kenangalem E, Lampah DA, Warikar N, Seal A, et al. Adverse pregnancy outcomes in an area where multidrug-resistant plasmodium vivax and plasmodium falciparum infections are endemic. Clin Infect Dis. 2008:46(9):1374-81.

71. Thompson JM, Eick SM, Dailey C, Dale AP, Mehta M, Nair A, et al. Relationship between pregnancy-associated malaria and adverse pregnancy outcomes: a systematic review and Meta-analysis. J Trop Pediatr. 2020;66(3):327-38.

72. Watson-Jones D, Weiss HA, Changalucha JM, Todd J, Gumodoka B, Bulmer J, et al. Adverse birth outcomes in United Republic of Tanzania: impact and prevention of maternal risk factors. Bull World Health Organ. 2007:85:9-18.

73. Andemel N, Gaoussou S, Barry A, Issiaka D, Mahamar A, Traore M, et al. Adverse pregnancy outcomes among women presenting at antenatal clinics in Ouélessébougou, Mali. Reprod Health. 2020;17(1):39.

74. Chico RM, Mayaud P, Ariti C, Mabey D, Ronsmans C, Chandramohan D. Prevalence of malaria and sexually transmitted and reproductive tract infections in pregnancy in sub-Saharan Africa: a systematic review. Jama. 2012;307(19):2079-86.

75. Masha SC, Wahome E, Vaneechoutte M, Cools P, Crucitti T, Sanders EJ. High prevalence of curable sexually transmitted infections among pregnant women in a rural county hospital in Kilifi, Kenya. PLoS One. 2017;12(3):e0175166

76. Warr AJ, Pintye J, Kinuthia J, Drake AL, Unger JA, McClelland RS, et al. Sexually transmitted infections during pregnancy and subsequent risk of stillbirth and infant mortality in Kenya: a prospective study. Sex Transm Infect. 2019;95(1):60-6.

77. Delnord M, Hindori-Mohangoo A, Smith L, Szamotulska K, Richards J, Deb-Rinker $\mathrm{P}$, et al. Variations in very preterm birth rates in 30 highincome countries: are valid international comparisons possible using routine data? BJOG Int J Obstet Gynaecol. 2017;124(5):785-94.

\section{Publisher's Note}

Springer Nature remains neutral with regard to jurisdictional claims in published maps and institutional affiliations. 